\title{
A novel combined level set model for automatic MR image segmentation
}

Jianzhang Li: Department of Orthopaedic Surgery, RWTH Aachen University Clinic, Pauwelsstraße 30, 52074, Aachen, Germany. E-mail: jli@ukaachen.de

Sven Nebelung: Institute of Diagnostic and Interventional Radiology, University Hospital Düsseldorf, Moorenstr. 5, 40225 Düsseldorf, Germany. E-mail: snebelung@ukaachen.de

Björn Rath: Department of Orthopaedic Surgery, Klinikum Wels-Grieskirchen, Grieskirchner Str. 42, 4600 Wels, Austria. E-mail: Bjoern.Rath@klinikum-wegr.at

Markus Tingart: Department of Orthopaedic Surgery, RWTH Aachen University Clinic, Pauwelsstraße 30, 52074, Aachen, Germany. E-mail: mtingart@ukaachen.de

Jörg Eschweiler: Department of Orthopaedic Surgery, RWTH Aachen University Clinic, Pauwelsstraße 30, 52074, Aachen, Germany. E-mail: joeschweiler@ukaachen.de

\section{Introduction}

Medical image processing comes along with object segmentation, which is one of the most important tasks in that field. Nevertheless, noise and intensity inhomogeneity challenge the segmentation procedure in magnetic resonance images.

\section{Methods}

The level set method has been widely used in object detection. The flexible integration of energy terms affords the level set method to deal with variable difficulties. In this paper, we introduce a novel combined level set model that mainly cooperates with an edge detector and a local region intensity descriptor. The noise and intensity inhomogeneities are eliminated by the local region intensity descriptor. The edge detector helps the level set model to locate the object boundaries more precisely.

\section{Results}

The proposed model was validated on synthesized images and magnetic resonance images of in vivo wrist bones. Comparing with the ground truth, the proposed method reached a Dice similarity coefficient of $>0.99$ on both image tests, while the compared segmentation approaches failed the segmentations.

\section{Conclusion}

The presented combined level set model can be used for the object segmentation in magnetic resonance images. 


\section{Investigating the influence of dielectric pads in $7 \mathrm{~T}$ magnetic resonance imaging - simulated and experimental assessment}

Maíra M. Garcia, General and Theoretical Electrical Engineering (ATE), University of Duisburg-Essen, and CENIDE - Center of Nanointegration Duisburg-Essen, D-47048 Duisburg, Germany, and Faculty of Electrical Engineering and Applied Natural Sciences, Westphalian University, Campus Gelsenkirchen, Germany, maira.martins-garcia@stud.uni-due.de

Maryam Vatanchi, Faculty of Electrical Engineering and Applied Natural Sciences, Westphalian University, Campus Gelsenkirchen, Germany, maryam.vatanchi@studmail.w-hs.de

Khallil T. Chaim, Department and Institute of Radiology, University of São Paulo, São Paulo, Brazil, khallil.chaim@hc.fm.usp.br

Maria C. G. Otaduy, Department and Institute of Radiology, University of São Paulo, São Paulo, Brazil, maria.otaduy@hc.fm.usp.br

Andreas Rennings, General and Theoretical Electrical Engineering (ATE), University of Duisburg-Essen, and CENIDE - Center of Nanointegration Duisburg-Essen, D-47048 Duisburg, Germany, andre.rennings@unidue.de

Daniel Erni, General and Theoretical Electrical Engineering (ATE), University of Duisburg-Essen, and CENIDE - Center of Nanointegration Duisburg-Essen, D-47048 Duisburg, Germany, daniel.erni@uni-due.de Waldemar Zylka, Faculty of Electrical Engineering and Applied Natural Sciences, Westphalian University, Campus Gelsenkirchen, Germany, waldemar.zylka@w-hs.de

\section{Introduction}

Dipole radiofrequency $(\mathrm{RF})$ elements have been successfully used to compose multi-channel radiofrequency coils for several different imaging targets, especially for ultra-high fields (UHF) magnetic resonance imaging $\left(B_{0} \geq 7 \mathrm{~T}\right)$. At UHF the magnetic $\left(B_{1}\right)$ components of RF fields can be very inhomogeneous, since the wavelength becomes comparable to the dimensions of the head. To improve the $\mathrm{B}_{1}$ efficiency and homogeneity, dielectric pads with high dielectric constants can be used as a passive $B_{1}$ shimmimg technique, because they induce a strong secondary magnetic field in their vicinity. Their use influence not only the $B_{1}$ field, but also the specific absorption rate (SAR) and consequently, the temperature distribution. To study these effects a $29 \mathrm{~cm}$-long transmission dipole RF coil element terminated by two meander and optimized dielectric pads were used for 7T MRI. Simulated and experimental results were obtained to analyse the $\mathrm{B}_{1}$ field distribution inside an agarose-gel based phantom for different configurations: with and without dielectric pads. Specific absoption rate (SAR) predictions were done to estimate energy absorption inside the object.

\section{Methods}

A single dipole RF element was used for RF signal transmission. A bottle filled with agarose-gel and dielectric pads (suspension of calcium titanate and deuterium oxide) were used for the experimental analysis. Numerical analysis simulating the same experimental setup were performed using the software Ansys.

\section{Results}

$B_{1}$ maps for the different configurations were acquired inside the phantom and compared with the simulations, indicating a good confidence in the simulation setup and revealing that dielectric pads improve $\mathrm{B}_{1}$ homogeneity distribution. Estimations of local SAR indicate a different distribution when using dielectric pads.

\section{Conclusion}

Numerical and experimental investigations indicate that dielectric pads improve $B_{1}$ homogeneity and address higher $\mathrm{B}_{1}$ magnitude for regions where it was previously not high enough. Simulations inform that local SAR distribution will change when using dielectric pads. 